\title{
ESTRUTURA E PADRÃO ESPACIAL DA VEGETAÇÃO EM UMA ÁREA DE CAATINGA
}

\author{
Gabriel Paes Marangon ${ }^{1}$, Rinaldo Luiz Caraciollo Ferreira² ${ }^{2}$ José Antônio Aleixo da Silva ${ }^{2}$, \\ David Fagner de Souza e Lira ${ }^{1}$, Emanuel Araújo Silva ${ }^{1}$, Gabrielle Hambrecht Loureiro ${ }^{3}$ \\ ${ }^{1}$ Eng. Florestal, Doutorando em Eng. Florestal, UFSM, Santa Maria, RS, Brasil - gabrimarangon@yahoo.com.br; \\ davidlira17@yahoo.com.br; mad_emanuel@hotmail.com.br \\ ${ }^{2}$ Eng. Florestal, Dr., Depto. de Ciência Florestal da UFRP, Recife, PE, Brasil - rinaldo@ dcfl.ufrpe.br; aleixo@dcfl.ufrpe.br \\ ${ }^{3}$ Eng $^{\text {a }}$ Florestal, Mestranda em Eng. Florestal, UFPR, Curitiba, PR, Brasil - ghloureiro@yahoo.com.br \\ Recebido para publicação: 02/07/2012 - Aceito para publicação: 05/02/2013
}

\begin{abstract}
Resumo
A vegetação do Nordeste revela carência relacionada ao manejo florestal da Caatinga. Objetivou-se estudar o padrão espacial de espécies e estrutura da vegetação em área de Caatinga, localizada no município de Floresta, PE. Em uma área de aproximadamente 10 ha, com histórico de perturbação, foram instaladas 20 parcelas de 20 × $20 \mathrm{~m}$, de forma sistemática. Todas as árvores com CAP a 1,30 m do solo $\geq 6 \mathrm{~cm}$ foram identificadas e tiveram suas circunferências e alturas mensuradas. Foram encontradas 18 espécies arbóreas, distribuídas em 15 gêneros, dentro de 6 famílias botânicas, com índice de diversidade de Shannon $\left(\mathrm{H}^{\prime}\right)$ de 2,11 nats.ind $^{-1}$, e área basal total estimada de 4,53 $\mathrm{m}^{2} \cdot \mathrm{ha}^{-1}$. Destacaram-se as famílias Fabaceae, com 48,93\% dos indivíduos, e Euphorbiaceae, com 41,28\%. As espécies Poincianella bracteosa (Tul.) L. P. Queiroz, Croton rhamnifolius Kunth, Manihot cartaginenses subsp. glaziovii (Muell. Arg.) Allem. e Mimosa ophtalmocentra Mart. ex Benth somaram 55\% do total do valor de importância. A maioria das espécies apresentou distribuição agregada, exceto algumas, como Myracrodruon urundeuva Fr. Allem. para o índice de McGuinnes (IGA), Commiphora leptophloeos (Mart.) J. B. Gillett para McGuinnes (IGA) e Payandeh (Pi) e Manihot cartaginenses subsp. glaziovii (Muell. Arg.) Allem. para Fracker e Brischle (Ki), que obtiveram tendência ao agrupamento.

Palavras-chave: Semiárido; Pernambuco; distribuição espacial.
\end{abstract}

\begin{abstract}
Structure and spatial pattern of vegetation in a caatinga area. Forest management deficiency in relation to Northeast vegetation, specifically caatinga, are remarkable. This research aims to analyze spatial patterns of species and vegetation structure in Caatinga area, located in the municipality of Floresta-PE. In an area of approximately 10 ha, and a history of disturbance, 20 plots $(20 \times 20 \mathrm{~m})$ were installed in systematic way. All trees with CAP (at $1.30 \mathrm{~m}$ above the ground) $\geq 6 \mathrm{~cm}$ had been identified, and their circumferences and heights measured. 18 tree species were sampled, distributed in 15 genus, within 6 botanical families, with diversity index of Shannon $\left(\mathrm{H}^{\prime}\right)$ of 2.11 nats / ind, and estimated total basal area of 4, $53 \mathrm{~m} 2$.ha-1. The highlighted families were Fabaceae, $48.93 \%$ of individuals, and Euphorbiaceae $41.28 \%$. The species Poincianella bracteosa (Tul.) L.P.Queiroz, Croton rhamnifolius Kunth, Manihot cartaginenses Subsp. glaziovii (Muell. Arg.) Allem., and Mimosa ophtalmocentra Mart. ex Benth totalized 55\% of the total value of importance. Most species revealed aggregated distribution, except some like Myracrodruon urundeuva Fr. Allem. for the McGuinness index (IGA), Commiphora leptophloeos (Mart.) J.B.Gillett for McGuinness (IGA) and Payandeh (Pi), and Manihot cartaginenses Subsp. glaziovii (Muell. Arg.) Allem. for Fracker and Brischle (Ki), which obtained grouping tendency.

Keywords: Semiarid; Pernambuco; spatial distribution.
\end{abstract}

\section{INTRODUÇÃO}

A Caatinga é a vegetação predominante na região Nordeste, cobrindo $54,53 \%$ dos $1.548 .672 \mathrm{~km}^{2}$ de área (IBGE, 2007). Está compreendida entre o paralelo de $3^{\circ} 17^{\prime} \mathrm{S}$ e o meridiano $35^{\circ} 45^{\prime} \mathrm{W}$, inserida no 
semiárido, envolvendo áreas dos estados do Ceará, Rio Grande do Norte, Paraíba, Pernambuco, Alagoas, Sergipe, sudoeste do Piauí e partes do interior da Bahia e do Norte de Minas Gerais (ANDRADE et al., 2005).

Pesquisas sobre a vegetação do Nordeste, em particular no estado de Pernambuco, ainda são muito carentes de resultados no que diz respeito ao manejo florestal sustentado da Caatinga. Esse bioma possui características restritas e peculiares que vêm confirmar sua exclusividade de ocorrência no território brasileiro. Essa vegetação recobre uma área mais ou menos contínua de clima quente e semiárido e apresenta plantas com características que as tornam totalmente adaptadas às condições da região: caducifólia, presença de herbáceas anuais, suculência, acúleos e espinhos, predominância arbustiva, árvores de pequeno porte, cobertura contínua de copas e espécies endêmicas (RODAL; SAMPAIO, 2002).

Para o conhecimento desse bioma, é necessária a utilização da fitossociologia como ferramenta, pois, por meio dela pode-se conhecer e realizar uma avaliação momentânea da frequência, densidade, dominância e diversidade de espécies que se encontram em uma floresta (SILVA, 2006).

O estudo de padrões de distribuição espacial é hoje uma das ferramentas mais utilizadas para entender o comportamento de diversos fenômenos (ANJOS et al., 1998). Nas ciências florestais, uma grande aplicação está nas investigações sobre a distribuição das espécies, principalmente daquelas ocorrentes em seu ambiente natural.

O estudo do padrão espacial de espécies que não tenham estudos detalhados permite gerar hipóteses sobre processos que dão origem a esse padrão (DALE, 1999). Por outro lado, o padrão pode ser resposta à heterogeneidade ambiental e às interações intra e interespecíficas, tais como competição e predação, dispersão, crescimento, senescência, mortalidade e reprodução (JANZEN, 1970; BAROT et al., 1999; DALE, 1999; PERRY et al., 2002; AIBA et al., 2004; PALMIOTTO et al., 2004; RODRIGUES et al., 2007).

Este trabalho objetivou analisar a estrutura e o padrão espacial de espécies de uma área de Caatinga, a fim de obter subsídios para o manejo florestal dessa região.

\section{MATERIAL E MÉTODOS}

\section{Caracterização da área de estudo}

A área de estudo está inserida na região fisiográfica do sertão do estado de Pernambuco. As parcelas foram alocadas na Fazenda Itapemirim, localizada no município de Floresta, PE (Figura 1), que está localizado a $433,4 \mathrm{~km}$ a oeste da cidade de Recife e encontra-se inserido, geologicamente, na Província Borborema, pertencente à unidade geoambiental da Depressão Sertaneja, que representa a paisagem típica do semiárido nordestino (COMPANHIA DE PESQUISA DE RECURSOS MINERAIS (CPRM), 2005).

A vegetação é basicamente composta por Caatinga hiperxerófila com trechos de floresta caducifólia. O clima é do tipo tropical semiárido, de acordo com a classificação de Köppen, apresentando-se como BSh', com precipitação média anual de $431,8 \mathrm{~mm}$ e temperatura média da ordem de $24,7^{\circ} \mathrm{C}$. A sede do município de Floresta se localiza a uma latitude $08^{\circ} 36^{\prime} 04^{\prime \prime}$ sul e a uma longitude de 38 $34^{\prime} 07^{\prime \prime}$ oeste, estando a uma altitude de 316 metros (CPRM, 2005). De acordo com a Empresa Brasileira de Pesquisa Agropecuária (EMBRAPA) (2006), o solo da região é o Luvissolo Crômico pouco profundo, com textura superficial arenosa a média.

O levantamento foi realizado em uma área de aproximadamente 10 ha, com histórico de perturbação, com evidência de exploração madeireira e pastagem de caprinos, observados em campo. Foram instaladas 20 parcelas $(20 \times 20 \mathrm{~m})$, de forma sistemática, distanciadas $80 \mathrm{~m}$ entre linhas e $80 \mathrm{~m}$ entre parcelas. Todas as árvores com circunferência a $1,30 \mathrm{~m}$ do solo (CAP) igual ou superior a $6,0 \mathrm{~cm}$ foram georreferenciadas, identificadas e mensuradas em relação ao CAP.

\section{Composição florística, diversidade e estrutura}

As parcelas foram georreferenciadas com GPS, num vértice previamente definido. Nas parcelas, todas as árvores mensuráveis foram identificadas individualmente através de uma plaqueta de PVC com numeração progressiva. Na identificação das espécies, foi utilizado o sistema de classificação de Angiosperm Phylogeny Group versão III (APG, 2009). 

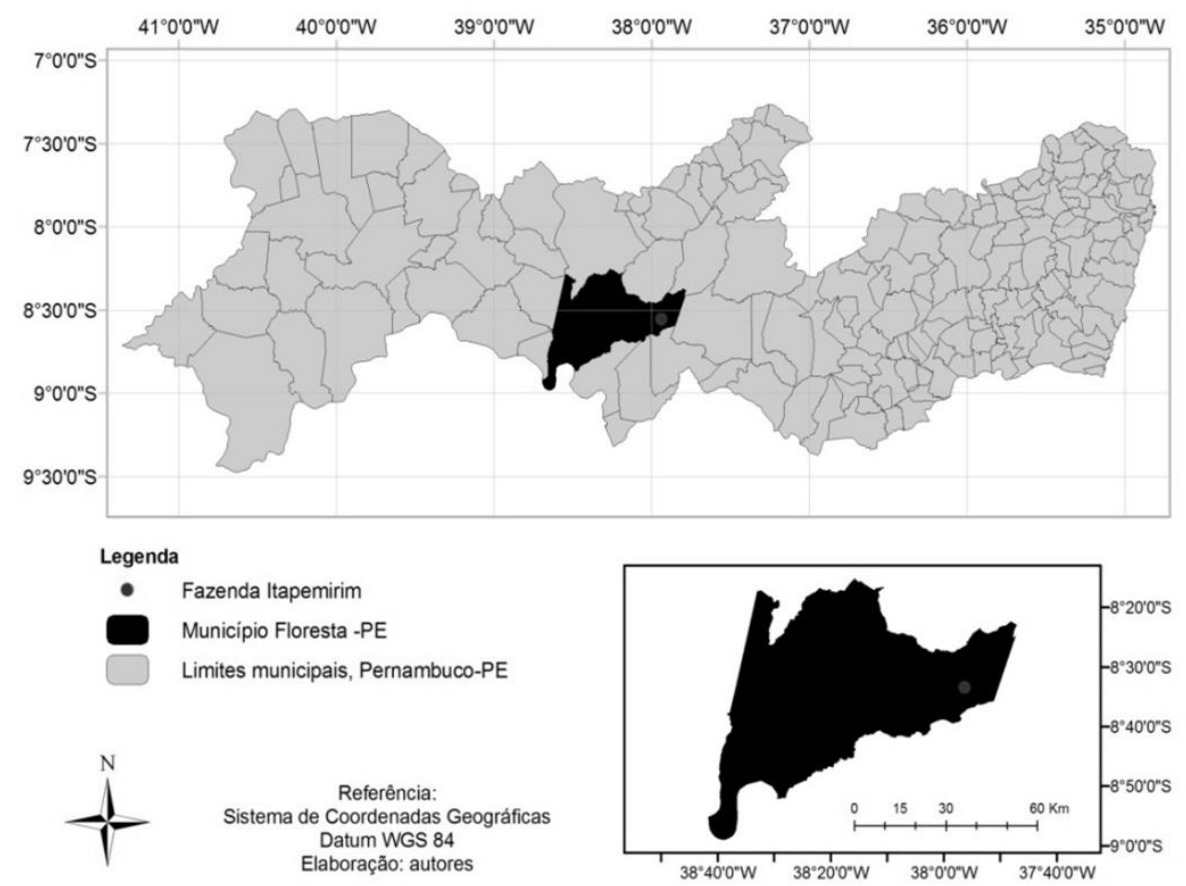

Figura 1. Esquema e mapa de localização do município e da fazenda Itapemirim, Floresta, PE. Figure 1. Layout and location map of the city and Itapemirim farm, Floresta, PE.

Em relação à diversidade florística, foram calculados os índices de Shannon $(\mathrm{H})$, Simpson $(\mathrm{C})$, Pielou (J) e coeficiente de mistura de Jentsch (QM), e para o estudo do padrão espacial das espécies dominantes (de maiores valores de importância), foi realizado primeiramente o estudo fitossociológico, por meio da análise da estrutura horizontal, com o qual se estimaram os parâmetros fitossociológicos de densidade, frequência e dominância (absoluta e relativa), valor de cobertura e valor de importância, conforme Muller-Dombois e Ellemberg (1974), utilizando-se o software Mata Nativa@ 2 (FUNDAÇÃO DE CIÊNCIA E TECNOLOGIA (CIENTEC), 2006).

As espécies foram distribuídas segundo classes de diâmetro, considerando uma amplitude de $3,0 \mathrm{~cm}$, apontada pelo software, a partir de um diâmetro mínimo de $1,9 \mathrm{~cm}$. Para o estudo da distribuição espacial das espécies, foram utilizados os índices de distribuição de Morisita, Payandeh, Fracker e Brischle e McGuinnes. A distribuição espacial das espécies se classifica obedecendo à seguinte escala: Morisita $\left(\mathrm{I}_{\delta}\right)-\mathrm{I}=1$ é distribuição ao acaso; $\mathrm{I}>1$ é do tipo agregada; $\mathrm{I}<1$ é distribuição regular; McGuinnes (IGA) - IGA $\mathrm{IG}_{\mathrm{i}} 1$ é distribuição uniforme, $\mathrm{IGA}_{\mathrm{i}}=1$ é distribuição aleatória, $1<\mathrm{IGA}_{\mathrm{i}} \leq 2$ é tendência ao agrupamento, $\mathrm{IGA}_{\mathrm{i}}>2$ é distribuição agregada ou agrupada; Fracker e Brischle (Ki) $-\mathrm{Ki} \leq$ 0,15 é distribuição aleatória, $0,15<\mathrm{Ki} \leq 1,0$ é tendência ao agrupamento, $\mathrm{Ki}>1$ é distribuição agregada ou agrupada; Payandeh $(\mathrm{Pi})-\mathrm{P}_{\mathrm{i}}<1$ é distribuição aleatória ou não agrupada, $1 \leq \mathrm{P}_{\mathrm{i}} \leq 1,5$ é tendência ao agrupamento, $\mathrm{P}_{\mathrm{i}}>1,5$ é distribuição agregada ou agrupada (CIENTEC, 2006).

\section{RESULTADOS E DISCUSSÃO}

\section{Análise florística}

Foram registradas 18 espécies arbóreas, distribuídas em 15 gêneros e seis famílias botânicas (Tabela 1), organizadas em ordem alfabética de família, gênero e espécie. Silva (2009), em estudo de Caatinga com histórico de diferentes usos no agreste pernambucano, encontrou 29 espécies, distribuídas em 22 gêneros e 17 famílias. Já Andrade et al. (2005), estudando áreas de Caatinga no Cariri paraibano, encontrou 16 espécies, 15 gêneros e sete famílias na área bem preservada, e seis espécies, seis gêneros e quatro famílias na degradada. Através disso foi possível identificar que essa área encontra-se dentro das variações em níveis florísticos existentes nos diferentes tipos de Caatinga. 
Em relação à riqueza de espécies, as famílias foram representadas da seguinte forma: Fabaceae, com sete espécies, Euphorbiaceae, com seis espécies, Anacardiaceae, com duas espécies, e Apocynaceae, Burseraceae e Caparaceae, com apenas uma espécie. Tais famílias ainda são citadas como sendo as de maior destaque em trabalhos realizados em Caatinga por Pereira et al. (2001) e Rodal et al. (1998), além de outros autores, como Andrade et al. (2005), citarem resultados semelhantes com predomínio dos mesmos táxons em áreas de Caatinga antropizadas e típicas.

Tabela 1. Listagem das espécies lenhosas, em ordem alfabética, de família, gênero e espécies encontradas em uma área de Caatinga da fazenda Itapemirim, município de Floresta, PE.

Table 1. List of woody species, in alphabetical order, of family, genus and species found in an area of Caatinga Itapemirim farm, municipality of Floresta, PE.

\begin{tabular}{lc}
\hline Família/espécie & Nome vulgar \\
\hline ANACARDIACEAE & \\
Myracrodruon urundeuva Allemão & Aroeira \\
Schinopsis brasiliensis Engl. & Baraúna \\
APOCYNACEAE & \\
Aspidosperma pyrifolium Mart. & Pereiro \\
BURSERACEAE & \\
Commiphora leptophloeos (Mart.) J.B. Gillett & Imburana-de-cambão \\
CAPARACEAE & Feijão-bravo \\
Cynophalla flexuosa L. J. Presl. & \\
EUPHORBIACEAE & \\
Cnidoscolus bahianus (Ule) Pax \& K. Hoffm. & Favela-brava \\
Cnidoscolus quercifolius Pohl & Faveleira \\
Croton rhamnifolius Willd. & Quebra-faca \\
Jatropha mollissima (Pohl) Baill. & Pinhão-bravo \\
Jatropha mutabilis (Pohl) Baill. & Pinhão \\
Manihot cartaginenses subsp. glaziovii (Muell. Arg.) Allem. & Maniçoba \\
FABACEAE & \\
Piptadenia stipulacea (Benth.) Ducke & jurema-branca \\
Anadenanthera colubrina var. cebil (Griseb.) Altschul & Angico \\
Bauhinia cheilanta (Bong). Steud. & Mororó \\
Mimosa ophtalmocentra Mart. ex Benth. & Jurema-de-embira \\
Mimosa tenuiflora (Willd.) Poir. & jurema-preta \\
Poincianella bracteosa (Tul). L. P. Queiroz & Catingueira \\
Senna spectabilis var. excelsa (Schad.) H. S. Irwin \& Barneby & Pau-de-besouro \\
\hline
\end{tabular}

As famílias Euphorbiaceae, Fabaceae e Apocynaceae compõem 96,53\% do total de indivíduos amostrados. Em estudo no semiárido da Paraíba, Carvalho et al. (2009) encontraram as famílias Euphorbiaceae, Fabaceae e Apocynaceae com 81,6\% dos indivíduos amostrados. Já Almeida Neto et al. (2009) encontraram as famílias Euphorbiaceae e Fabaceae com 87,64\% do total de indivíduos amostrados, salientando que sua área de estudo apresentou baixa riqueza quando comparada a outros trabalhos, mas esclarece tal fator devido à baixa pluviosidade de $300 \mathrm{~mm}$.

Entende-se, diante do exposto, que existe concentração de espécies em reduzido número de famílias, fato corriqueiro em vegetação de Caatinga, e que, no presente estudo, demonstra as áreas com pouca riqueza de espécies. Isso revela que na presente área existe predominância de poucas espécies, mas com elas ocorrendo em elevado número de indivíduos, que sobrepujam o ambiente de estudo.

\section{Diversidade florística}

Em relação à diversidade florística, os índices gerais se comportaram da seguinte forma: Shannon: $\mathrm{H}^{\prime}=2,11$ nats.ind ${ }^{-1}$; Simpson: $\mathrm{C}=0,84$; Pielou: $\mathrm{J}^{\prime}=0,73$; coeficiente de mistura de Jentsch: $\mathrm{QM}=0,02$.

Silva (2009) encontrou, no agreste pernambucano, valores similares para uma área abandonada há cerca de 30 anos: $\mathrm{H}^{\prime}=2,46$ nats.ind $^{-1} ; \mathrm{C}=0,73 ; \mathrm{J}^{\prime}=0,88$; e $\mathrm{QM}=0,03$. 
Calixto Júnior et al. (2011) obtiveram $\mathrm{H}^{\prime}$ de 1,39 nats.ind ${ }^{-1}$ e $\mathrm{J}^{\prime}$ de 0,50 , acerca dos quais os autores comentam que são valores de acordo com outros estudos realizados em ambientes de Caatinga, que variam de 1,10 a 3,09 nats.ind ${ }^{-1}$. Embora considerado baixo, os autores justificam fazendo menção ao histórico de intervenção antrópica da área. Observa-se, dessa forma, que os resultados encontrados no presente estudo para os índices de diversidade são compatíveis com outros estudos de Caatinga.

Em geral, estudos em Caatinga não demonstram diversidades muito elevadas, sendo que, no presente estudo, evidenciou-se pouca diversidade, até mesmo abaixo de outros estudos em áreas de Caatinga. Esse fato pode ser explicado por fatores principalmente no que diz respeito ao solo com perfil pequeno, além de alta pedregosidade, predominância de espécies com alta resistência e fatores relacionados a históricos de perturbação da área.

\section{Estrutura da vegetação}

Em relação aos parâmetros fitossociológicos, foram amostrados os valores reais de 981 indivíduos vivos distribuídos em 0,8 ha, ocasionando uma área basal de 3,63 $\mathrm{m}^{2}$.ha ${ }^{-1}$. Em relação aos mesmos valores, mas estimados de acordo com o programa Mata Nativa $\odot$ 2, encontraram-se 1.227 indivíduos.ha ${ }^{-1}$ e 4,53 $\mathrm{m}^{2} \cdot \mathrm{ha}^{-1}$ de área basal, com altura média, máxima e mínima de 3,63, 10 e $1,5 \mathrm{~m}$, respectivamente. Os DAP médio, mínimo e máximo foram de 4,0, 1,90 e $20,08 \mathrm{~cm}$, respectivamente. Percebe-se que, apesar do elevado número de indivíduos, que eles apresentaram áreas basais bastante reduzidas, configurando a ocorrência de plantas com baixos valores de diâmetros.

Destacam-se as dez espécies para o valor de importância com seus respectivos valores em percentagem (Tabela 2): Poincianella bracteosa (19,94\%), Croton rhamnifolius (15,36\%), Manihot cartaginenses subsp. glaziovii (10,83\%), Mimosa ophtalmocentra (8,89\%), Piptadenia stipulacea (7,06\%), Aspidosperma pyrifolium (6,25\%), Myracrodruon urundeuva (6,06\%), Commiphora leptophloeos (5,03\%), Cnidoscolus bahianus (3,96\%) e Bauhinia cheilanta (3,81\%). Dessa forma, apenas as espécies $P$. bracteosa, $C$. rhamnifolius, $M$. cartaginenses e $M$. ophtalmocentra concentraram mais de $55,02 \%$ do referido valor de importância.

Das espécies com maior densidade relativa, merecem destaque Croton rhamnifolius, com 27,12\%, Poincianella bracteosa (24,06\%), Mimosa ophtalmocentra (11,11\%) e Manihot cartaginenses subsp. glaziovii (9,58\%), que contribuíram com mais de $70 \%$ em relação a esse parâmetro na área em estudo (Figura 1), devido aos seus elevados números de indivíduos por hectare (333, 295, 137 e 118 ind.ha $^{-1}$, respectivamente). Oliveira et al. (2009) encontraram, em estudo fitossociológico em determinada área do Cariri paraibano, apenas quatro espécies concentrando 55,22\% do VI e 67,55\% do VC, sendo elas Poincianella bracteosa, Aspidosperma pyrifolium, Croton sonderianus e Bauhinia cheilantha. Diante disso, percebe-se que apenas quatro espécies, como em outros estudos de diferentes fisionomias de Caatinga, demonstraram mais da metade da importância relacionada à área de estudo. (MARACAJÁ et al., 2003; FABRICANTE; ANDRADE, 2008; ANDRADE et al., 2007; RODAL et al., 2008).

Diante da análise da comunidade, constatou-se que existe grande concentração de indivíduos nas duas primeiras classes de diâmetro, existindo um maior número de indivíduos (701) na primeira classe de diâmetro $(1,9$ a $4,9 \mathrm{~cm})$ e 325 para a segunda classe $(4,9$ a $7,9 \mathrm{~cm})$, juntos contribuindo com $83 \%$ dos indivíduos amostrados em toda a área de estudo. Nas demais classes, percebe-se uma queda bastante acentuada, à medida que há um aumento nas classes de diâmetro, apresentando uma curva exponencial em forma de "J" invertido na sua distribuição diamétrica (Figura 2).

Analisando a figura 2, entende-se que a comunidade estudada pode se encontrar em estágio de recuperação, por existir predomínio dos indivíduos nas primeiras classes de diâmetro, com $61 \%$ dos indivíduos de Poincianella bracteosa e Croton rhamnifolius concentrados nas duas primeiras classes. Essas espécies predominam na formação da tipologia da área. No local existe evidência de uma forte ação antrópica, com exploração madeireira e pastagem de caprinos. Esses impactos podem influenciar no estágio sucessional.

Almeida Neto et al. (2009), em estudo no semiárido paraibano, obtiveram distribuição diamétrica representada na forma de $\mathrm{J}$ invertido, em que 2690 indivíduos distribuíram-se nas três primeiras classes e 1975 indivíduos foram distribuídos somente na primeira classe, com queda acentuada para as classes seguintes, corroborando as distribuições do presente trabalho. 
Tabela 2. Parâmetros fitossociológicos para os indivíduos com $\mathrm{CAP} \geq 6 \mathrm{~cm}$, em uma área de Caatinga, Floresta, PE, em ordem decrescente de espécies de maior valor de importância (VI). AB: área basal; DA: densidade absoluta; DR: densidade relativa; FA: frequência absoluta; FR: frequência relativa; DoA: dominância absoluta; DoR: dominância relativa; VC: valor de cobertura; VI: valor de importância.

Table 2. Phytosociological parameters for individuals with CAP $\geq 6 \mathrm{~cm}$ in an area of Caatinga, Floresta, $\mathrm{PE}$, in decreasing order of species importance value (VI). AB: basal area; DA: density absolute; DR: relative density; FA: absolute frequency; FR: relative frequency; DoA: absolute dominance; DoR: relative dominance; VC: margin calls; VI: importance value.

\begin{tabular}{lrrrrrrrrr}
\hline Nome científico & AB & DA & DR & FA & FR & DoA & DoR & VC(\%) & VI (\%) \\
\hline Poincianella bracteosa & 0.8642 & 295,00 & 24,06 & 85 & 11,97 & 1,080 & 23,80 & 23,93 & 19,94 \\
Croton rhamnifolius & 0,3304 & 332,50 & 27,12 & 70 & 9,86 & 0,413 & 9,10 & 18,11 & 15,36 \\
Manihot cartaginenses & & & & & & & & & \\
subsp. glaziovii & 0,3975 & 117,50 & 9,58 & 85 & 11,97 & 0,497 & 10,95 & 10,27 & 10,83 \\
Mimosa ophtalmocentra & 0,2842 & 136,25 & 11,11 & 55 & 7,75 & 0,355 & 7,83 & 9,47 & 8,89 \\
Piptadenia stipulacea & 0,1334 & 93,75 & 7,65 & 70 & 9,86 & 0,167 & 3,68 & 5,66 & 7,06 \\
Aspidosperma pyrifolium & 0,2722 & 77,50 & 6,32 & 35 & 4,93 & 0,340 & 7,50 & 6,91 & 6,25 \\
Myracrodruon urundeuva & 0,3527 & 26,25 & 2,14 & 45 & 6,34 & 0,441 & 9,71 & 5,93 & 6,06 \\
Commiphora leptophloeos & 0,3906 & 10,00 & 0,82 & 25 & 3,52 & 0,488 & 10,76 & 5,79 & 5,03 \\
Cnidoscolus bahianus & 0,0938 & 36,25 & 2,96 & 45 & 6,34 & 0,117 & 2,58 & 2,77 & 3,96 \\
Bauhinia cheilanta & 0,0334 & 42,50 & 3,47 & 50 & 7,04 & 0,042 & 0,92 & 2,19 & 3,81 \\
Anadenanthera colubrina & 0,1278 & 16,25 & 1,33 & 35 & 4,93 & 0,160 & 3,52 & 2,42 & 3,26 \\
Schinopsis brasiliensis & 0,2095 & 5,00 & 0,41 & 20 & 2,82 & 0,262 & 5,77 & 3,09 & 3,00 \\
Mimosa tenuiflora & 0,0983 & 15,00 & 1,22 & 20 & 2,82 & 0,123 & 2,71 & 1,97 & 2,25 \\
Jatropha mollissima & 0,0086 & 7,50 & 0,61 & 25 & 3,52 & 0,011 & 0,24 & 0,42 & 1,46 \\
Jatropha mutabilis & 0,0067 & 6,25 & 0,51 & 20 & 2,82 & 0,008 & 0,18 & 0,35 & 1,17 \\
Cnidoscolus quercifolius & 0,0257 & 6,25 & 0,51 & 15 & 2,11 & 0,032 & 0,71 & 0,61 & 1,11 \\
Cynophalla flexuosa & 0,0013 & 1,25 & 0,10 & 5,0 & 0,70 & 0,002 & 0,04 & 0,07 & 0,28 \\
Senna spectabilis & 0,0003 & 1,25 & 0,10 & 5,0 & 0,70 & 0.000 & 0,01 & 0,06 & 0,27 \\
\hline Total & 3,6305 & 1226.25 & 100 & 710 & 100 & 4,538 & 100 & 100 & 100 \\
\hline & & & & & & & & &
\end{tabular}

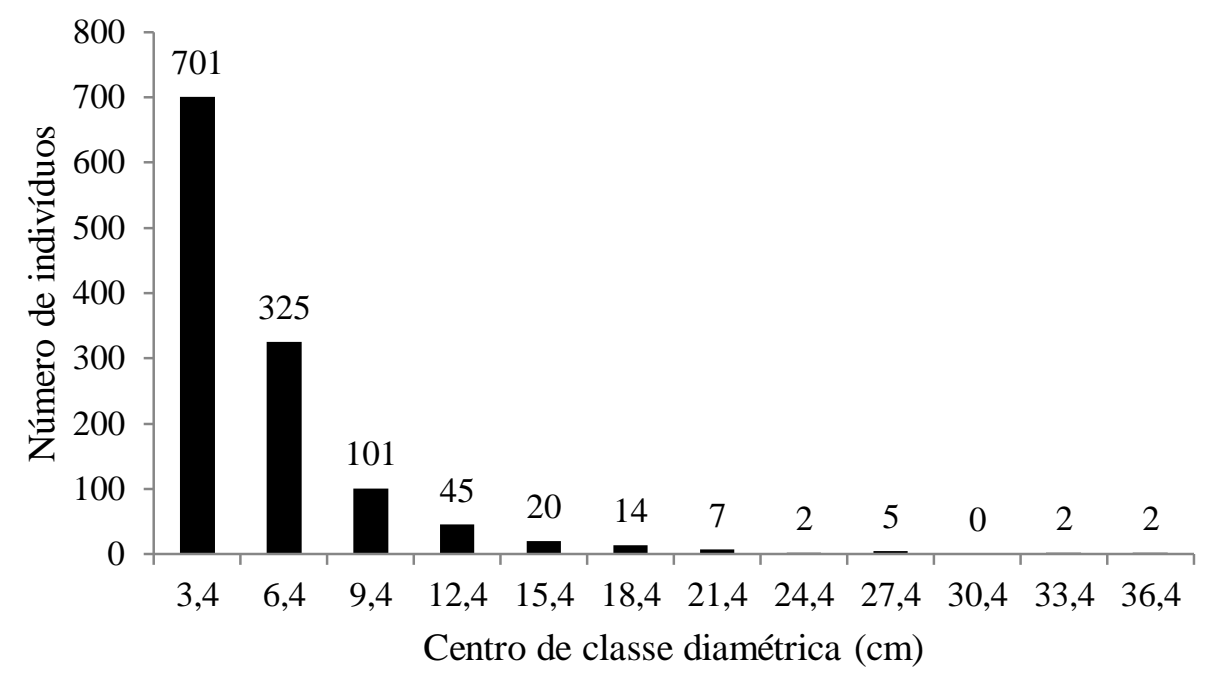

Figura 2. Distribuição diamétrica da vegetação em uma área de Caatinga, Floresta, PE, expressa em número de indivíduos por hectare por centro de classes de diâmetro, com amplitude de classe de $3 \mathrm{~cm}$.

Figure 2. Vegetation diameter distribution in an area of Caatinga, Floresta, PE, expressed in number of individuals per hectare in the center of diameter classes, with amplitude class of $3 \mathrm{~cm}$. 


\section{Padrão espacial}

Ao analisar o nível de agregação das espécies considerando os diferentes índices (Tabela 3), foram calculados os índices de dispersão de Morisita $\left(\mathrm{I}_{\delta}\right)$, Payandeh $(\mathrm{Pi})$, Fracker e Brischle $(\mathrm{Ki})$ e McGuinnes (IGA) para a área em estudo.

Tabela 3. Padrão espacial das dez espécies florestais lenhosas com maiores valores de importância (VI) em uma área de Caatinga, Floresta, PE, onde os índices de agregação de Morisita (I $\delta$ ), McGuinnes (IGA), Fracker e Brischle (Ki) e Payandeh (Pi), por espécie e número de unidades amostrais em que a espécie ocorre $(\mathrm{N})$, são representados.

Table 3. Spatial pattern of ten hardwood tree species with highest importance values (IV) in an area of Caatinga, Floresta-PE, where are represented the following aggregation index Morisita (I $\delta$ ), McGuinness (IGA), and Frack Brischle (Ki) and Payandeh (Pi) by species and number of plots where the species occurs $(\mathrm{N})$.

\begin{tabular}{lccccc}
\hline Nome científico & N & $\mathbf{I}_{\boldsymbol{\delta}}$ & IGA & Ki & Pi \\
\hline Poincianella bracteosa & 17 & 2,66 & 6,22 & 2,75 & 17,06 \\
Croton rhamnifolius & 14 & 2,16 & 11,05 & 8,34 & 16,81 \\
Manihot cartaginenses subsp. glaziovii & 17 & 1,44 & 2,48 & $\underline{0,78}$ & 3,72 \\
Mimosa ophtalmocentra & 11 & 2,61 & 6,83 & 7,3 & 10,36 \\
Piptadenia stipulacea & 14 & 2,4 & 3,11 & 1,76 & 6,06 \\
Aspidosperma pyrifolium & 7 & 3,67 & 7,2 & 14,38 & 7,91 \\
Myracrodruon urundeuva & 9 & 2,48 & $\underline{1,76}$ & 1,27 & 2,05 \\
Commiphora leptophloeos & 5 & 5,29 & $\underline{1,39}$ & 1,36 & $\underline{1,42}$ \\
Cnidoscolus bahianus & 9 & 4,17 & 2,43 & 2,38 & 3,01 \\
Bauhinia cheilanta & 10 & 4,66 & 2,45 & 2,1 & 9,54 \\
\hline
\end{tabular}

As dez espécies de maior VI apresentaram padrão de agregação conforme o índice de Morisita. Já o índice de McGuinnes (IGA) refletiu o padrão agregado para a grande maioria das espécies, exceto para Myracrodruon urundeuva e Commiphora leptophloeos, que apresentaram tendência ao agrupamento, sendo que tais espécies possuem vinte e cinco indivíduos distribuídos em nove parcelas e dez indivíduos distribuídos em cinco parcelas, respectivamente. Possivelmente, devido a esse reduzido número de indivíduos em relação ao total e devido ao índice ser calculado levando em consideração parâmetros de densidade, explica-se tal tendência ao agrupamento.

De acordo com o índice de Fracker e Brischle, todas as espécies se comportaram com padrão de agregação, exceto Manihot cartaginenses subsp. glaziovii, que obteve tendência ao agrupamento, destoando assim dos demais resultados, em que ela apresentou índices agregados. Por fim, pela classificação de Payandeh, a exceção foi Commiphora leptophloeos, também com tendência ao agrupamento com o restante das espécies, oferecendo padrão agregado. Mendes Júnior et al. (2009), verificando o padrão de distribuição espacial das espécies de Caatinga e utilizando os índices de agregação de McGuinnes (IGA), Fracker e Brischle (Ki) e o de Payandeh (Pi), relatam que a maioria das espécies apresentam, pelos três índices utilizados, distribuição agregada, e comenta que isso se deve a uma estratégia da Caatinga de se estabelecer com grande quantidade de indivíduos em áreas que sofreram alguma perturbação.

Sabendo-se que a área de estudo apresenta fatores morfológicos, como os mecanismos de dispersão das espécies a curta distância, fatores ambientais, como solos rasos e distúrbios naturais, além dos fatores fitossociológicos, como competição inter e intraespecífica, e levando em consideração que tais categorias propiciam uma agregação das espécies, entendemos de certo modo as distribuições das espécies em sua maioria. Murphy e Lugo (1986) comentam que padrões agregados são comuns para todas as florestas tropicais e acrescentam que tais padrões também são encontrados em florestas tropicais secas.

\section{CONCLUSÕES}

- A formação vegetal se encontra na tentativa de recuperação e percebe-se um estágio inicial de sucessão, devido à mesma ser altamente perturbada, com área basal reduzida, caracterizada por espécies de caráter pioneiro, em razão da ação antrópica e dos históricos de uso relacionados à área. 
- Para os padrões espaciais da área, a grande maioria das espécies apresentou distribuição agregada, porém as espécies Myracrodruon urundeuva, Commiphora leptophloeos e Manihot cartaginenses subsp. glaziovii obtiveram tendência ao agrupamento. Dessa forma, como em geral nos estudos relacionados à distribuição espacial em outros biomas, as espécies da Caatinga também apresentam distribuições agregadas, fato que contribui ao entendimento do comportamento das referidas espécies analisadas e colabora para possíveis intervenções, sejam elas de caráter exploratório ou conservacionista, em áreas de Caatinga.

\section{REFERÊNCIAS}

AIBA, S. I.; KANEHIRO, K.; TAKYU, M. Habitat associations with topography and canopy structure of tree species in a tropical montane forest on Mount Kinabalu, Borneo. Plant Ecology, Washington. v. 174, n. 1, p. 147 - 161, 2004.

ALMEIDA NETO, J. X.; ANDRADE, A. P., LACERDA, A. V.; FELIX, L. P.; BRUNO, R. L. A. Composição florística, estrutura e análise populacional do feijão-bravo (Capparis flexuosa L.) no semiárido paraibano, Brasil. Caatinga, Mossoró. v. 22, n. 4, p. 187 - 194, 2009.

ANDRADE, L. A.; OLIVEIRA, F. X.; NEVES C. M. L.; FELIX L. P. Análise da vegetação sucessional em campos abandonados no agreste paraibano. Revista Brasileira de Ciências Agrárias, Recife. v. 2, n. 2, p. $135-142,2007$.

ANDRADE, L. A.; PEREIRA, I. M.; LEITE, U. T.; BARBOSA, M. R. V. Análise da cobertura de duas fisionomias de caatinga com diferentes históricos de uso, no município de São João do Cariri, estado da Paraíba. Cerne, Lavras v. 11, n. 3, p. 253 - 262, 2005.

ANGIOSPERM PHYLOGENY GROUP III. An update of the Angiosperm Phylogeny Group classification for the orders and families of flowering plants. Botanical Journal of the Linnean Society. London, v. 16, [s.n], p. 105 - 121, 2009.

ANJOS, A.; COUTO, H. T. Z; BATISTA, J. L. F.; REIS, A. Análise de um efeito de um manejo em regime de rendimento sustentável sobre o padrão de distribuição espacial do palmiteiro (Euterpe edulis Martius), utilizando a função K de Ripley. Revista Árvore, Viçosa. v. 22, n. 2, p. 215 - 225, 1998.

BAROT, S.; GIGNOUX, J.; MENAUT, J. Demography of a savanna palm tree: predictions from comprehensive spatial pattern analyses. Ecology, Washington. v. 80, p. 1987 - 2005, 1999.

CALIXTO JÚNIOR, J. T.; DRUMOND, M. A.; ALVES JÚNIOR, F. T. Estrutura fitossociológica de um fragmento de caatinga sensu stricto 30 anos após corte raso, Petrolina, PE, Brasil. Caatinga, Mossoró. v. 24, n. 2, p. 67 - 74, 2011.

CARVALHO, E. C. D.; SOUZA. B. C.; TROVÃO. D. M. B. M. Grupos ecológicos em vegetação de caatinga no semiárido da Paraíba, Brasil. In: IX Congresso de Ecologia do Brasil, 13 a 17 de setembro de 2009, São Lourenço, MG. Anais... São Lourenço. 2009, p. 82 - 100.

COMPANHIA DE PESQUISA DE RECURSOS MINERAIS (CPRM) - Serviço Geológico do Brasil. Projeto cadastro de fontes de abastecimento por água subterrânea. Diagnóstico do município de Ibimirim, estado de Pernambuco/Organizado [por] MASCARENHAS J. C. et. al. Recife: CPRM/PRODEEM, 2005. 33 p. Disponível em <http://www.cprm.gov.br acesso em 14 de maio de 2010>. Acesso em: 3/10/2010.

DALE, M. R. T. Spatial pattern analysis in plant ecology. Cambridge University Press. Cambridge. 1999. $326 \mathrm{p}$.

EMPRESA BRASILEIRA DE PESQUISA AGROPECUÁRIA (EMBRAPA). Sistema brasileiro de classificação de solos. Rio de Janeiro: EMBRAPA, 2. ed. 2006, 412 p.

FABRICANTE, J. R.; ANDRADE, L. A. Análise estrutural de um remanescente de caatinga no Seridó paraibano. Oecologia Brasiliensis, Rio de Janeiro. v. 11, n. 3, p. 341 - 349, 2008.

FUNDAÇÃo DE CIÊNCIA E TECNOLOGIA (CIENTEC). Mata Nativa 2: Manual do usuário. Viçosa, MG: CIENTEC: 2006. 
INSTITUTO BRASILEIRO DE GEOGRAFIA E ESTATÍSTICA (IBGE). Mapas de biomas e vegetação. Disponível em:< http:// www.ibge.gov.br>. Acesso em: 20/07/2007.

JANZEN, D. H. Herbivores and the number of tree species in tropical forests. The American Naturalist, Chicago. v. 104, n. 940, p. 501 - 528, 1970.

MARACAJÁ, P. B.; BATISTA, C. H. F.; SOUSA, A. H.; VASCONCELOS, W. E. Levantamento florístico e fitossociológico do extrato arbustivo-arbóreo de dois ambientes na Vila Santa Catarina, Serra do Mel, RN. Revista de Biologia e Ciências da Terra, Belo Horizonte, MG, v. 3, n. 2, p. 20 - 33, 2003.

MENDES JÚNIOR, I. J. H; FERREIRA, R. L. C.; SILVA, J. A. A.; ALVES JÚNIOR, F. T.; SILVA, A. E.; CÉSPEDES, G. H. G. Distribuição espacial, diversidade florística e regeneração de uma área de caatinga após 20 anos de corte. In: IX JORNADA DE ENSINO, PESQUISA E EXTENSAO, 2009, Recife. Anais... Recife: EDUFRPE, 2009. v. 9.

MÜLLER-DOMBOIS, D.; ELLENBERG, H. Aims and methods of vegetation ecology. New York: Willey \& Sons, 1974, 547 p.

MURPHY, P. G.; LUGO, A. E. Ecology of tropical dry forest. Annual Review of Ecology and Systematics, v. 17, p. 67 - 88. 1986.

OLIVEIRA, P. T. B.; TROVÃO, D. M. B. M.; CARVALHO, E. C. D.; SOUZA, B. C.; FERREIRA L. M. R. Florística e fitossociologia de quatro remanescentes vegetacionais em área de serra no Cariri paraibano. Caatinga, Mossoró. v. 22, n. 4, p. 169 - 178, 2009.

PALMIOTTO, P. A.; DAVIES, S. J.; VOGT, K. A.; ASHTON, M.; VOGT, D. J.; ASHTON, P. S. Soilrelated habitat specialization in dipterocarp rain forest tree species in Borneo. Journal of Ecology, London. v. 92, n. 4, p. 609 - 623, 2004.

PEREIRA, I. M.; ANDRADE, L. A.; COSTA, J. R. M.; DIAS, J. M. Regeneração natural em um remanescente de caatinga sob diferentes níveis de perturbação, no Agreste Paraibano. Acta Botanica Brasilica, São Paulo. v. 15, n. 3, p. 413 - 426, 2001.

PERRY, J. N.; LIEBHOLD, A. M.; ROSENBERG, M. S. J.; DUNGAN, M.; MIRITI, A.; JAKOMULSKA, S. Illustrations and guidelines for selecting statistical methods for quantifying spatial pattern in ecological data. Ecography, Lound. v. 25, n. 5, p. 578 - 600, 2002.

RODAL, M. J. N.; ANDRADE, K. V. A.; SALES, M. F.; GOMES, A. P. S. Fitossociologia do componente lenhoso de um refúgio vegetacional no município de Buíque, Pernambuco. Revista Brasileira de Biologia, São Carlos. v. 58, n. 3, p. 517 - 526, 1998.

RODAL, M. J. N.; COSTA, K. C. C.; SILVA, A. C. B. L. Estrutura da vegetação caducifólia espinhosa (Caatinga) de uma área do sertão central de Pernambuco. Hoehnea, São Paulo. v. 35, p. 209 - 217, 2008.

RODAL, M. J. N.; SAMPAIO, E. V. S. B. A vegetação do bioma caatinga, p. 11 - 24. In: SAMPAIO, E. V. S. B., GIULIETTI, A. M.; VIRGINIO, J.; GAMARRA-ROJAS, C. F. L. (eds.). Vegetação e Flora de Caatinga. Recife: APNE, 2002. 176 p.

RODRIGUES, L. A.; CARVALHO, D. A.; OLIVEIRA FILHO, A. T.; CURI, N. Efeitos de solos e topografia sobre a distribuição de espécies arbóreas em um fragmento de Floresta Estacional Semidecidual, em Luminárias, MG. Revista Árvore, Viçosa. v. 31, n. 1, p. 25 - 35, 2007.

SILVA, S. O. Estudo de duas áreas de vegetação da caatinga com diferentes históricos de uso no agreste pernambucano. 2009. 83 f. Dissertação (Mestrado em Ciências Florestais) - Universidade Federal Rural de Pernambuco, Recife, PE.

SILVA, W. C. Estudo da regeneração natural de espécies arbóreas em quatro fragmentos de Floresta Ombrófila Densa no município de Catende, zona da mata sul de Pernambuco. 2006. $71 \mathrm{f}$. Dissertação (Mestrado em Ciências Florestais) - Universidade Federal Rural de Pernambuco, Recife, PE. 
